\title{
Erratum to: Introduction to the Physics of Silicene and other 2D Materials
}

\author{
Seymur Cahangirov, Hasan Sahin, Guy Le Lay, Angel Rubio
}

(C) Springer International Publishing AG 2017

S. Cahangirov et al., Introduction to the Physics of Silicene and other 2D Materials, Lecture Notes in Physics 930,

DOI 10.1007/978-3-319-46572-2

DOI 10.1007/978-3-319-46572-2_7

The affiliation for the editor Angel Rubio was incorrect. The correct affiliation is:

Max Planck Institute for the Structure and Dynamics of Matter

Hamburg

Germany

Universidad del País Vasco

San Sebastián

Spain

The updated online version of the original book can be found at

http://dx.doi.org/10.1007/978-3-319-46572-2 